\title{
Photoacoustic endoscopy probe using a coherent fibre-optic bundle
}

\author{
R. Ansari, E. Zhang, S. Mathews, A. E. Desjardins, and P. C. Beard \\ Department of Medical Physics and Biomedical Engineering, University College London, \\ Gower Street, WC1E 6BT, UK
}

\begin{abstract}
There is considerable interest in the development of photoacoustic endoscopy probes (PAE) for applications in foetal medicine, interventional surgery and gastroenterology. However, most previous PAE probes employ a combination of mechanical scanning and piezoelectric transducers at the distal end which can be technically complex and pose challenges in achieving the required level of miniaturisation and acoustic performance. To overcome these limitations, we present two novel all-optical forward-viewing endoscopic probes that use coherent fibre bundles to address a Fabry-Pérot polymer film ultrasound sensor.
\end{abstract}

Keywords: Photoacoustic tomography, photoacoustic endoscopy, Coherent fibre bundle, Image bundle, FabryPerot sensors.

\section{INTRODUCTION}

Photoacoustic endoscopy (PAE) has many potential medical applications including guiding interventional procedures in foetal medicine or laparoscopic surgery and assessing pathologies in the gastrointestinal tract. Most previous PAE probes integrate mechanical scanners and piezoelectric transducers at the distal end to acquire $2 \mathrm{D}$ or $3 \mathrm{D}$ images. ${ }^{1-4}$ In this study, we present two novel, all-optical forward-viewing endoscopic probes that employ a different approach in which a coherent fibre bundle is used to optically address different spatial points on a Fabry-Pérot (FP) polymer film ultrasound sensor located at the distal end of the bundle. This method avoids the need for mechanical scanning at the distal end of the probe, and the FP sensor offers advantages in terms of acoustic performance and practicality over piezoelectric equivalents. ${ }^{5-8}$ These advantages include wide bandwidth (tens of $\mathrm{MHz}$ ) for high resolution endoscopic imaging, high sensitivity with acoustically small element sizes as required for PA imaging in tomography mode, optical transparency for backward mode operation, and inexpensive fabrication.
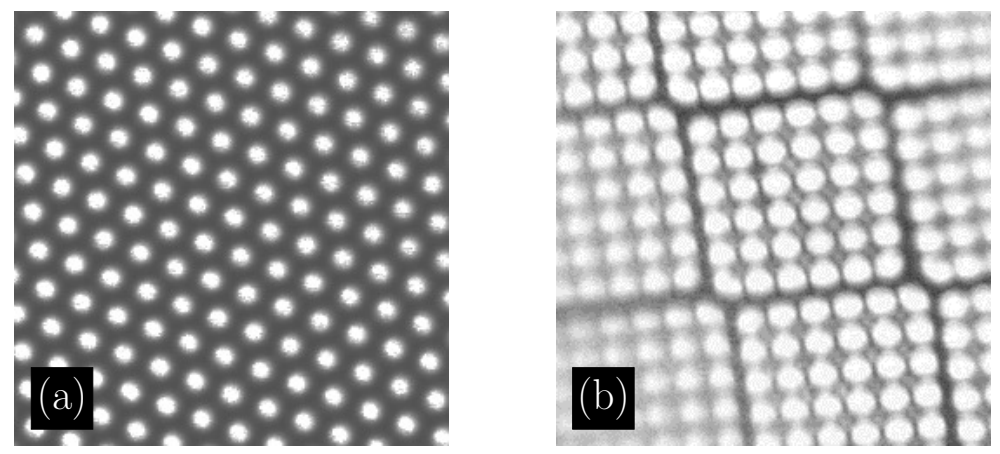

Figure 1. Image of (a) rigid, and (b) flexible coherent fibre bundles end faces under broadband illumination. The core diameters are $12 \mu \mathrm{m}$ and $10 \mu \mathrm{m}$ respectively.

Opto-Acoustic Methods and Applications in Biophotonics II, edited by Vasilis Ntziachristos, Roger Zemp, Proc. of SPIE Vol. 9539, 953905 - ( 2015 SPIE · CCC code: 1605-7422/15/\$18 - doi: 10.1117/12.2183820 


\section{EXPERIMENTAL SET-UP}

Two PAE probes, one rigid and the other flexible, were developed using commercially available coherent fibre bundles. The rigid PAE probe employed an $8 \mathrm{~cm}$ long image conduit $(0.55 \mathrm{NA} ; 10 \mu \mathrm{m}$ core diameter), which has 50,419 cores within a $3.2 \mathrm{~mm}$ outer diameter [Figure 1(a)]. A Fabry-Pérot (FP) sensor, comprising a $12 \mu \mathrm{m}$ thick Parylene C film sandwiched between $85 \%$ \& $98 \%$ reflective Au mirrors, was deposited on to the end face of the rigid fibre bundle (see Figure 2). The acoustic bandwidth of this sensor is approximately $45 \mathrm{MHz}$. Using the experimental set-up shown in Figure 2, the proximal end of the bundle was optically scanned along a line with a CW wavelength-tunable interrogation laser beam $(1550 \mathrm{~nm}$ center wavelength) in order to address different spatial points on the FP sensor. This probe was evaluated by mapping the output of two PZT ultrasound transducers: a planar $15 \mathrm{MHz}$ transducer and a focused $20 \mathrm{MHz}$ transducer.

The second PAE probe employed a $1 \mathrm{~m}$ long flexible wound fibre bundle $(0.63 \mathrm{NA}, 10 \mu \mathrm{m}$ core diameter), which has 161,604 cores packed into $4 \mathrm{~mm} \times 4 \mathrm{~mm}$ aperture. The image of the end-face in Figure 1(b) shows the arrangement of the cores in the wound fibre bundle. A similar experimental system to that shown in Figure 2 was used to optically scan the proximal end of the bundle in order to interrogate the FP sensor at the distal end. The FP sensor was fabricated by depositing a $20 \mu \mathrm{m}$ Parylene C film in between two dielectric mirrors; its acoustic bandwidth was approximately $40 \mathrm{MHz}$. The dielectric mirrors were designed to be highly transmissive to excitation wavelengths for backward-mode photoacoustic imaging. This probe was evaluated by mapping the photoacoustic signals generated in absorbing targets and reconstructing an image from the measured data using a time-reversal reconstruction algorithm. ${ }^{9}$ A fibre-coupled Q-switched Nd:YAG laser was used as the excitation source, which provided $8 \mathrm{~ns}$ pulses with $45 \mathrm{~mJ}$ of pulse energy at $20 \mathrm{~Hz}$ repetition rate.

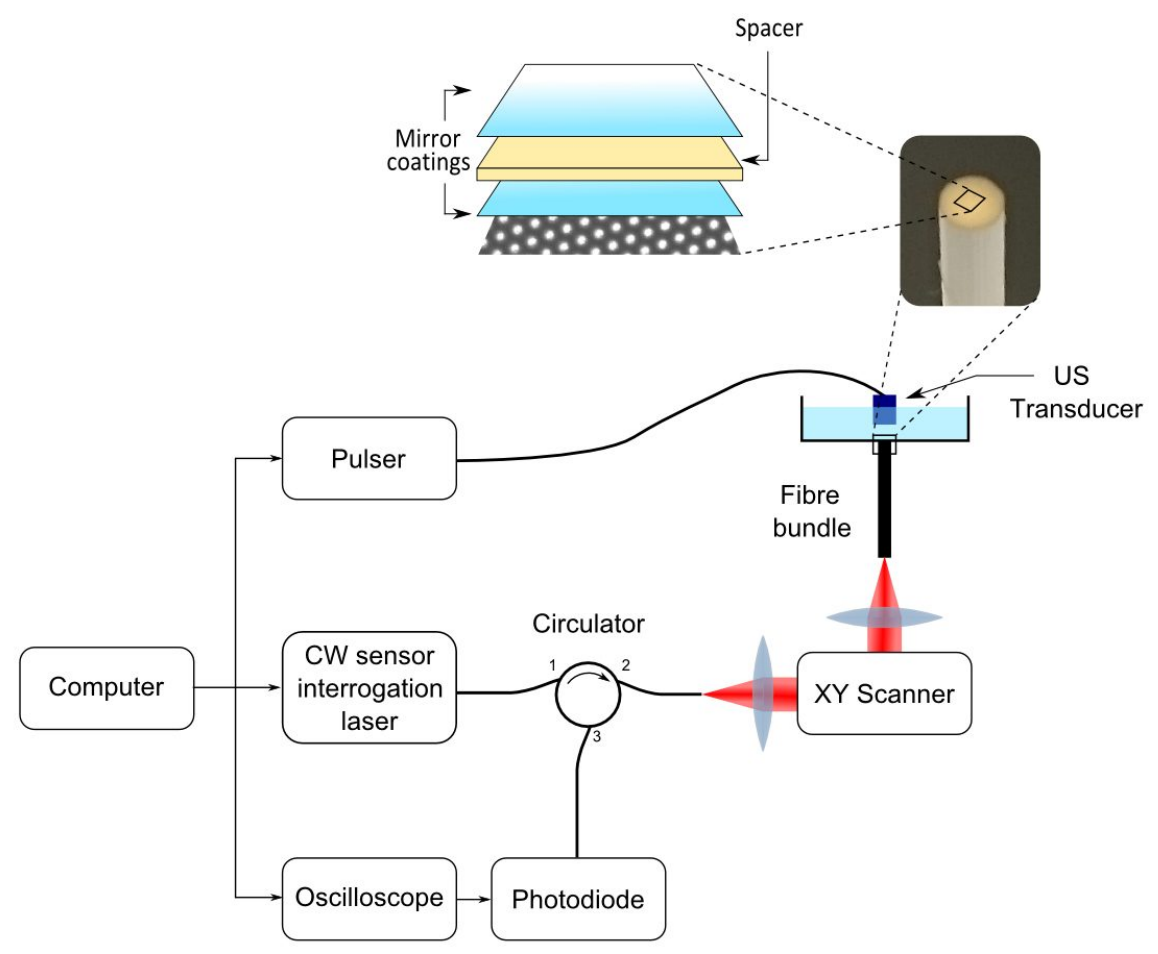

Figure 2. Experimental set-up of the PAE system. The close-up shows a schematic of the structure of the Fabry-Pérot ultrasonic sensor and a photograph of the end-face of the $3.2 \mathrm{~mm}$ diameter rigid coherent fibre bundle. 


\section{RESULTS AND DISCUSSION}

\subsection{Rigid PAE probe}

The interferometer transfer function (ITF) of the Fabry-Pérot sensor, deposited on the distal end face of the rigid PAE probe is shown in Figure 2(a). In addition to the two primary fringes, high frequency oscillations can also be seen. These are likely due to crosstalk and a consequence of the very tightly packed nature of the cores (Fig 1), which results in wavelength dependent coupling between adjacent cores. In this example, the ITF was low-pass filtered (shown as the red trace in Figure 3(a)) to remove the oscillations. This allows for an accurate representation of the ITF to be recovered, which is required to accurately set the optimum bias wavelength (the wavelength that corresponds to the maximum slope of the ITF and yields maximum sensitivity). To demonstrate the feasibility of imaging ultrasonic waves at frequencies representative of photoacoustic signals, the output of two PZT transducers were mapped by scanning the interrogation beam along a line of length $2.5 \mathrm{~mm}$ at the proximal end of the bundle. Figure 3(a) shows the acoustic field distribution from a $20 \mathrm{MHz}$ planar transducer, and Figure 3(b) shows the mapped acoustic field from a $15 \mathrm{MHz}$ focused ultrasound transducer.

(a)

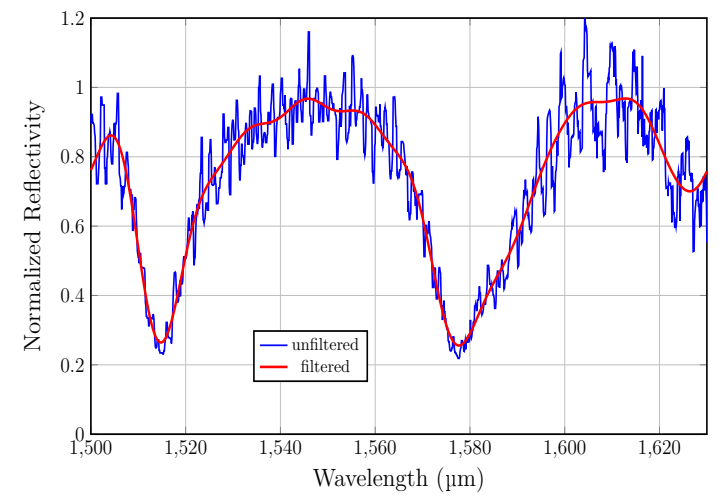

(b)

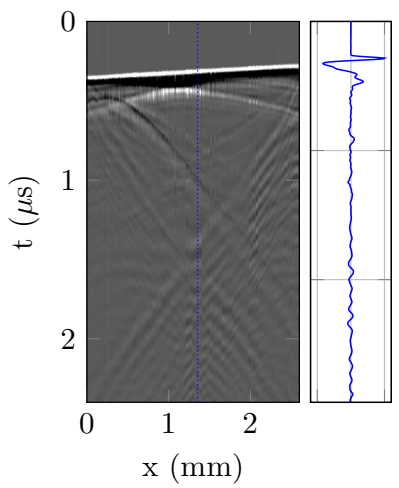

(c)

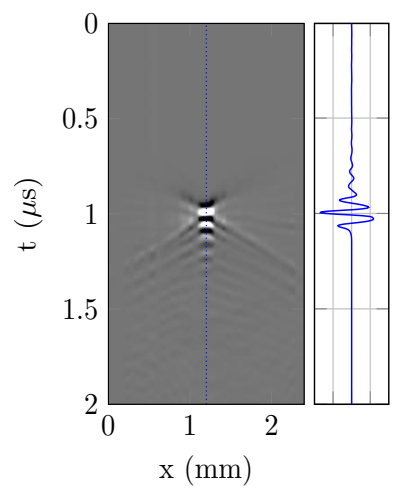

Figure 3. (a) Interferometer transfer function of the Fabry-Pérot ultrasound sensor at the distal end of the rigid PAE probe. (b) Acoustic field mapped using rigid PAE probe from $20 \mathrm{MHz}$ planar transducer, and (c) $15 \mathrm{MHz}$ focused transducer.

\subsection{Flexible PAE probe}

The experiments described in the previous section demonstrate the feasibility of optically addressing different spatial points on an FP ultrasound sensor located at the tip of a rigid coherent bundle. Although a rigid probe is suitable for some minimally invasive applications, many applications require a flexible imaging probe that can be inserted into the working channel of a flexible endoscope. The flexible fibre bundle described in section 2 was therefore used to address the FP sensor and photoacoustic images of a ribbon and leaf skeleton phantom were acquired. The schematic in Figure 4(a) shows the backward-mode illumination of the sample with the excitation light, and the arrangement of absorbing ribbons in a water tank. The map of measured photoacoustic signals, $\mathrm{P}(\mathrm{x}, \mathrm{t})$ (where $\mathrm{x}$ and $\mathrm{t}$ are lateral and axial dimensions, respectively), from the ribbon phantom is shown in Figure 4(b). A time reversal algorithm was used to reconstruct the photoacoustic image as shown in Figure 4(c). ${ }^{9}$ The axial line spread function is $33 \mu \mathrm{m}$, which was determined by taking the FWHM of a profile in the z-direction through the reconstructed ribbon feature as shown in Figure 4(d). A profile in the x-direction through the same feature was also acquired and the FWHM found to be $270 \mu \mathrm{m}$. This is likely to be an underestimate of the lateral resolution as the relatively large lateral dimensions of the ribbon means that it does not represent a spatial delta function in the $\mathrm{x}$-direction. Previous measurements suggest the lateral resolution at this depth should be approximately $100 \mu \mathrm{m} .^{8}$

The three dimensional photoacoustic imaging capability of a flexible PAE probe was demonstrated by imaging a leaf skeleton, which was coated in India ink. Figure 5 (a) shows the $5 \mathrm{~mm} \times 5 \mathrm{~mm}$ section of the leaf skeleton which was imaged and the corresponding $\mathrm{x}-\mathrm{y}$ maximum intensity projection of the reconstructed 3D photoacoustic 


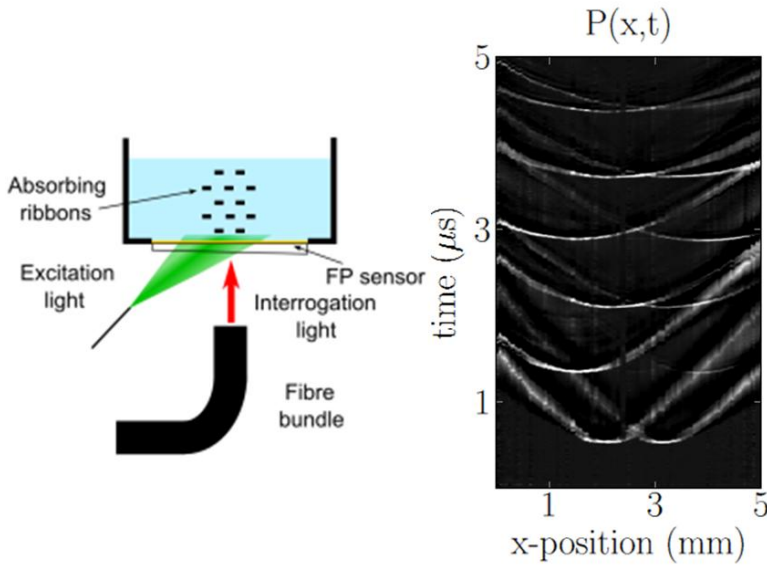

(a)

(b)

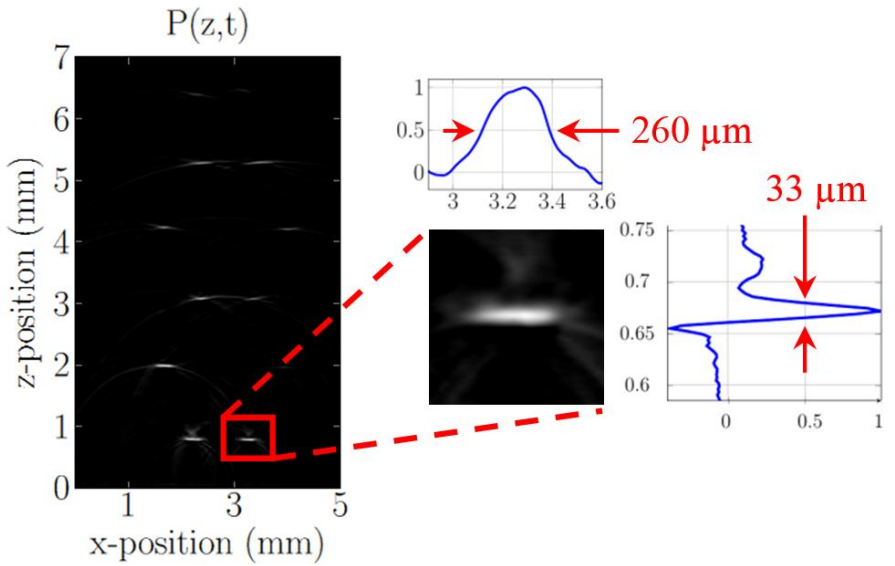

(c) (d)

Figure 4. Distal end of flexible PAE probe and ribbon phantom (a). Measured acoustic field (b) and reconstructed photoacoustic image of the ribbon phantom (c). The step size of the line-scan was $40 \mu \mathrm{m}$. Expanded view of a ribbon feature along with horizontal and vertical profiles through its center (d).

image is shown in Figure 5(b). The central vein of the leaf skeleton and its two branches are clearly visualized. Because of coarse lateral sampling, in steps of $100 \mu \mathrm{m}$, finer structures of the skeleton are not visible.
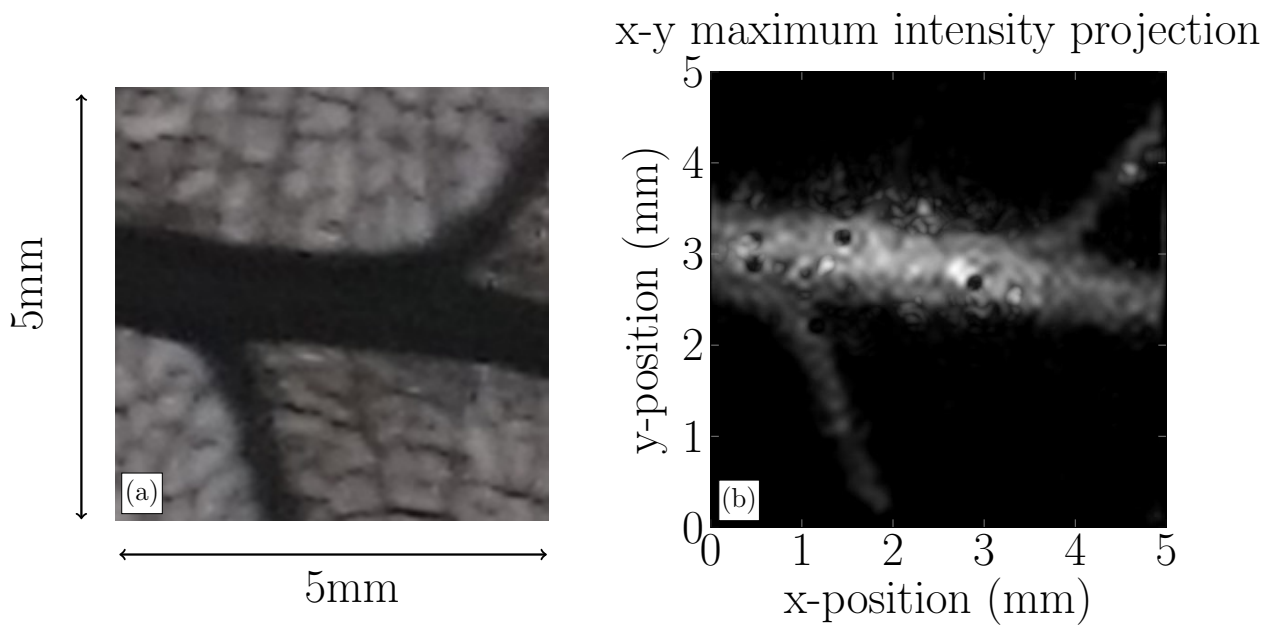

Figure 5. Photoacoustic image of a leaf phantom obtained using flexible PAE probe, (a) photograph of the leaf skeleton phantom. (b) $x-y$ maximum intensity projection of the reconstructed 3D photoacoustic image of the same area shown in (a).

\section{CONCLUSIONS}

Two novel forward-viewing PAE probes were developed and evaluated using rigid and flexible coherent fibre bundles with FP ultrasound sensors located at their distal ends. This preliminary study has demonstrated the feasibility of (i) addressing different spatial points on an FP ultrasound sensor using an optically scanned coherent fibre bundle; (ii) mapping acoustic waves at ultrasonic frequencies representative of photoacoustic signals at the distal end of a bundle; (iii) acquiring 2D and 3D photoacoustic images of phantoms using a flexible probe. This new approach offers several advantages over previous distal-end scanning PAE probes such as no moving parts at the distal end, relatively simple and inexpensive fabrication and the potential for wideband sensitive acoustic 
performance. Future work will focus on optimising sensitivity, bandwidth, and engineering a practical probe for in vivo endoscopic use.

\section{ACKNOWLEDGMENTS}

The authors acknowledge support from Wellcome Trust Innovation Fund, UK and European Union project FAMOS (FP7 ICT, Contract 317744).

\section{REFERENCES}

[1] Yang, J.-M., Maslov, K., Yang, H.-C., Zhou, Q., Shung, K. K., and Wang, L. V., "Photoacoustic endoscopy," Opt. Lett. 34, 1591-1593 (May 2009).

[2] Yang, J.-M., Chen, R., Favazza, C., Yao, J., Li, C., Hu, Z., Zhou, Q., Shung, K. K., and Wang, L. V., "A 2.5-mm diameter probe for photoacoustic and ultrasonic endoscopy," Opt. Express 20, 23944-23953 (Oct 2012).

[3] Yang, J.-M., Favazza, C., Chen, R., Yao, J., Cai, X., Maslov, K., Zhou, Q., Shung, K. K., and Wang, L. V., "Simultaneous functional photoacoustic and ultrasonic endoscopy of internal organs in vivo," Nat. Med. 18, 1297-1303 (August 2012).

[4] Dong, B., Chen, S., Zhang, Z., Sun, C., and Zhang, H. F., "Photoacoustic probe using a microring resonator ultrasonic sensor for endoscopic applications," Opt. Lett. 39(15), 4372-75 (2014).

[5] Zhang, E., Laufer, J., and Beard, P., "Backward-mode multiwavelength photoacoustic scanner using a planar fabry-perot polymer film ultrasound sensor for high-resolution three-dimensional imaging of biological tissues," Appl. Opt. 47(4), 561-77 (2008).

[6] Laufer, J., Norris, F., Cleary, J., Zhang, E., Treeby, B., Cox, B., Johnson, P., Scambler, P., Lythgoe, M., and Beard, P., "In vivo photoacoustic imaging of mouse embryos," J. Biomed. Opt. 17(6), 061220 (2012).

[7] Zhang, E. Z., Laufer, J. G., Pedley, R. B., and Beard, P. C., "In vivo high-resolution 3d photoacoustic imaging of superficial vascular anatomy," Phys. Med. Biol. 54(4), 1035-46 (2009).

[8] Laufer, J., Johnson, P., Zhang, E., Treeby, B., Cox, B., Pedley, B., and Beard, P., "In vivo preclinical photoacoustic imaging of tumor vasculature development and therapy," J. Biomed. Opt. 17(5), 056016 (2012).

[9] Treeby, B. E., Zhang, E., and Cox, B. T., "Photoacoustic tomography in absorbing acoustic media using time reversal," Inverse Problems 26, 115003 (2010). 